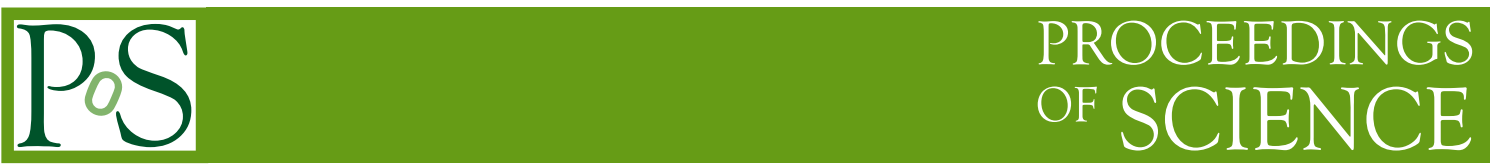

\title{
Global fit to right-handed neutrino mixing at 1 loop
}

\author{
Josu Hernandez-Garcia* \\ Departamento de Física Teórica, Universidad Autónoma de Madrid, Cantoblanco E-28049 \\ Madrid, Spain. \\ Instituto de Física Teórica UAM/CSIC, Calle Nicolás Cabrera 13-15, Cantoblanco E-28049 \\ Madrid, Spain. \\ E-mail: josu.hernandez@uam.es
}

We extend the Standard Model with 3 right-handed neutrinos with an approximate lepton number symmetry, which leads to sizable neutrino mixing while generating the neutrino masses and PMNS matrix measured in oscillation experiments. Constraints on the right-handed neutrino mixing are derived through a global fit to electroweak precision observables. We analyze explicitly the impact of one-loop corrections on these constraints.

The European Physical Society Conference on High Energy Physics

22-29 July 2015

Vienna, Austria

${ }^{*}$ Speaker. 


\section{Introduction}

The origin of the observed pattern of neutrino masses and mixings in neutrino oscillation experiments (see e.g. Ref. [1] for a recent summary) comprises one of the few experimental evidences for physics beyond the Standard Model (SM) of particle physics. The simplest and most popular extension to account for these experimental observations consists in the addition of right-handed neutrinos to the SM particle content. Given their singlet nature, a Majorana mass term for the right-handed neutrinos is directly allowed in the Lagrangian, thus inducing a new mass scale -the only one unrelated to electroweak (EW) symmetry breaking- to be determined by data.

The smallness of neutrino masses could derive from symmetry arguments [2, 3, 4, 5]. Indeed, the Weinberg operator [6] leading to neutrino masses in Seesaw mechanisms is protected by the $L$ symmetry, conserved in the SM and violated in two units by the Weinberg operator. Thus, if the pattern of the Yukawa couplings and Majorana masses in a Seesaw realization is such that it conserves $L$, the Weinberg operator will never be generated and the SM neutrinos will remain massless, even for $Y_{V} \sim 1$ and Majorana masses of the order of the EW scale. Small violations of $L$ in this pattern would thus induce the small neutrino masses observed in oscillation experiments. In this class of models fall the popular inverse [2,3] or linear [7] Seesaw mechanisms.

It was recently pointed out in [8] that loop corrections involving the extra heavy neutrinos played an important role when deriving such constraints on heavy-active neutrino mixing, obtaining qualitatively different results to those derived by staying at leading order. In particular, good fits with relatively large heavy-active mixing were found in [8], since it is possible to alleviate the tension in some observables through the partial cancellation of the tree and loop level contributions encoded in the $T$ parameter. However, when Ref. [9] also investigated the relevance of the $T$ parameter the same cancellation was not reproduced and in [10] it was argued that loop contributions should always be negligible.

In this work we clarify the role of loop contributions to the determination of the heavy-active neutrino mixing. We have computed the one-loop diagrams contributing to the observables and the analytical expressions together with the full numerical results can be found in our main article [11].

\section{The parametrization}

We will restrict our study to the extension of the SM by 3 right-handed neutrino fields. Thus, the full $6 \times 6$ mixing matrix $U$ is the unitary matrix that diagonalizes the extended neutrino mass matrix that can be written as [12]:

$$
U=\left(\begin{array}{cc}
\sum_{n=0}^{\infty} \frac{\left(-\Theta^{\dagger}\right)^{n}}{(2 n) !} & \sum_{n=0}^{\infty} \frac{\left(-\Theta^{\dagger}\right)^{n}}{(2 n+1) !} \Theta \\
-\sum_{n=0}^{\infty} \frac{\left(-\Theta^{\dagger} \Theta\right)^{n}}{(2 n+1) !} \Theta^{\dagger} & \sum_{n=0}^{\infty} \frac{\left(-\Theta^{\dagger} \Theta\right)^{n}}{2 n !}
\end{array}\right)\left(\begin{array}{cc}
U_{\mathrm{PMNS}} & 0 \\
0 & I
\end{array}\right),
$$

and where the general matrix $\Theta$ represents the mixing between active $\left(v_{e}, v_{\mu}\right.$ and $\left.v_{\tau}\right)$ and heavy $\left(N_{1}, N_{2}\right.$ and $\left.N_{3}\right)$ neutrino states, and the mass eigenstates $m$ and $M$. 
Sizable phenomenology requires both $M_{i} \sim O\left(\Lambda_{\mathrm{EW}}\right)$ and $Y_{N} \sim O(1)$ while keeping small light neutrino masses. Thus, an approximate lepton number symmetry $L$ is needed. The only mass matrices that conserve $L$ and leads to three heavy massive neutrinos are [13, 14]:

$$
m_{D}=\frac{\nu_{\mathrm{EW}}}{\sqrt{2}}\left(\begin{array}{ccc}
Y_{e} & Y_{\mu} & Y_{\tau} \\
\varepsilon_{1} Y_{e}^{\prime} & \varepsilon_{1} Y_{\mu}^{\prime} & \varepsilon_{1} Y_{\tau}^{\prime} \\
\varepsilon_{2} Y_{e}^{\prime \prime} & \varepsilon_{2} Y_{\mu}^{\prime \prime} & \varepsilon_{2} Y_{\tau}^{\prime \prime}
\end{array}\right) \quad \text { and } \quad M_{N}=\left(\begin{array}{ccc}
\mu_{1} & \Lambda & \mu_{3} \\
\Lambda & \mu_{2} & \mu_{4} \\
\mu_{3} & \mu_{4} & \Lambda^{\prime}
\end{array}\right)
$$

with all $\varepsilon_{i}$ and $\mu_{j}$ small lepton number violating parameters. Indeed, setting all $\varepsilon_{i}=0$ and $\mu_{j}=0$, $L$ is recovered with the following lepton number assignments $L_{e}=L_{\mu}=L_{\tau}=L_{1}=-L_{2}=1$ and $L_{3}=0$. Then, in the $L$-conserving limit, this leads to 3 massless light neutrinos, a heavy Dirac pair $M_{1}=M_{2}=\Lambda$ and a heavy decoupled Majorana singlet $M_{3}=\Lambda^{\prime}$, but an arbitrarily large mixing:

$$
\Theta=\frac{v_{\mathrm{EW}}}{2 \Lambda}\left(\begin{array}{ccc}
-i Y_{e}^{*} & Y_{e}^{*} & 0 \\
-i Y_{\mu}^{*} & Y_{\mu}^{*} & 0 \\
-i Y_{\tau}^{*} & Y_{\tau}^{*} & 0
\end{array}\right) \equiv \frac{1}{\sqrt{2}}\left(\begin{array}{ccc}
-i \theta_{e} & \theta_{e} & 0 \\
-i \theta_{\mu} & \theta_{\mu} & 0 \\
-i \theta_{\tau} & \theta_{\tau} & 0
\end{array}\right)
$$

Fixing neutrino oscillation data [1], one of the three elements of Eq. (2.3) can be expressed in terms of the other two, the light neutrino scale and the PMNS matrix. Thus, in our numerical exploration of the parameter space in Section 4 we will consider the 9 free parameters of Table 1.

\begin{tabular}{|c||c|c|c|c|c|}
\hline Parameter & $\left|Y_{e}\right| \times\left|Y_{\mu}\right|$ & $\left|Y_{e}\right|-\left|Y_{\mu}\right|$ & $m_{1}[\mathrm{eV}]$ & $\Lambda[\mathrm{GeV}]$ & Phases: $\alpha_{e}, \alpha_{\mu}, \delta, \alpha_{1} \& \alpha_{2}$ \\
\hline Range & $\left(0,10^{-4}\right)$ & $(-0.1,0.1)$ & $\left(10^{-5}, 1\right)$ & $\left(10^{3}, 10^{4}\right)$ & $(0,2 \pi)$ \\
\hline
\end{tabular}

Table 1: The 9 free parameters of our scan: the modulus and phase of the electron and muon Yukawas $\left|Y_{e}\right|$, $\left|Y_{\mu}\right|, \alpha_{e}$ and $\alpha_{\mu}$, the Majorana mass scale $\Lambda$, the absolute light neutrino mass $m_{1}$ and the 3 yet unknown CP-violation phases (Dirac and Majorana) in the PMNS mixing matrix: $\delta, \alpha_{1}$ and $\alpha_{2}$.

\section{Observables}

We have chosen the most representative set of observables that contains the dominant constraints and the main effects pointed out in [8]. The loop amplitudes of the processes have been computed exploiting the Goldstone-boson equivalence theorem [15] under the assumption that the mass of the extra neutrinos $M_{i}$ is larger than the gauge boson masses; i.e. $M_{i}>M_{W, Z}$. Thus, we have made the simplifying assumption that the most relevant loop corrections are those were the loops are mediated by either the Higgs boson, $h$, the Goldstone bosons $\phi^{ \pm}$and $\phi^{0}$ or the heavy Majorana neutrinos. The set of 13 independent observables analyzed in this study is composed of: 8 ratios constraining electroweak universality: $R_{\mu e}^{\pi}, R_{\tau \mu}^{\pi}, R_{\mu e}^{W}, R_{\tau \mu}^{W}, R_{\mu e}^{K}, R_{\tau \mu}^{K}, R_{\mu e}^{l}, R_{\tau \mu}^{l}$; the invisible $Z$ width; the $W$ mass $M_{W}$; and the 3 rare flavour-changing decays: $\mu \rightarrow e \gamma, \tau \rightarrow \mu \gamma$ and $\tau \rightarrow e \gamma$.

All observables are determined as a function of the three most precise EW measurements: $\alpha$, $M_{Z}$ and $G_{\mu}\left(G_{F}\right.$ as measured from $\mu$ decay) [16] and recibe contributions from the loop corrections to the $W$ and $Z$ boson propagators. These new contributions modify the tree level expression of the observables in such a way that the common correction is typically given by $1-\left|\theta_{e}\right|^{2}-\left|\theta_{\mu}\right|^{2}-$ $2 \alpha T$. This common dependence on the tree level and oblique corrections $T$ is the source of the cancellation analyzed in Ref. [8]. 


\section{Results}

\subsection{Constraints form the global fit}

With the 13 observables discussed in Section 3 we build a $\chi^{2}$ function depending on the 9 parameters listed in Table 1. We make use of Markov chain Monte Carlo (MCMC) techniques for efficient parameter exploration. The range in which the 9 free parameters are varied is also summarized in Table 1.

In Fig. 1 we show the results of our MCMC scan for the 2 degrees of freedom constraints of different combinations of the heavy-active mixings $\theta_{\alpha}$ defined in Eq. (2.3). The contours correspond to the $1 \sigma, 90 \%$ and $2 \sigma$ frequentist confidence regions.
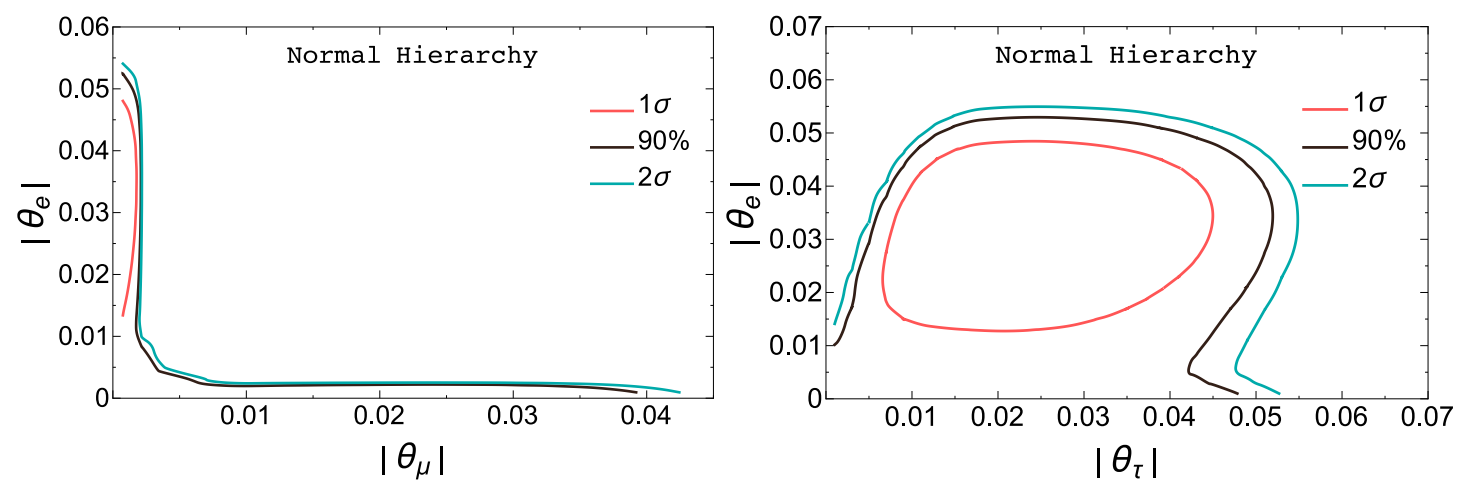

Figure 1: Contours for $\theta_{e}, \theta_{\mu}$ and $\theta_{\tau}$ at $1 \sigma$ (red), $90 \%$ CL (black) and $2 \sigma$ (blue) for normal hierarchy.

The limits of the 1 and $2 \sigma$ regions for the three mixing parameters are summarized in Table 2.

\begin{tabular}{|c|c|c|c|c|c|}
\hline \multicolumn{2}{|c|}{$\theta_{e}$} & \multicolumn{2}{c|}{$\theta_{\mu}$} & \multicolumn{2}{c|}{$\theta_{\tau}$} \\
\hline $1 \sigma$ & $2 \sigma$ & $1 \sigma$ & $2 \sigma$ & $1 \sigma$ & $2 \sigma$ \\
\hline $0.034_{-0.014}^{+0.009}$ & $<0.050$ & $<3.2 \cdot 10^{-4}$ & $<0.037$ & $0.018_{-0.013}^{+0.019}$ & $<0.049$ \\
\hline
\end{tabular}

Table 2: Constraints on $\theta_{e}, \theta_{\mu}$, and $\theta_{\tau}$ for normal hierarchy.

We have performed a second set of MCMC runs where all loop corrections have been removed. The results of these simulations are identical to the ones stemming from the full computation.

\subsection{The $T$ parameter}

The leading contributions (not suppressed by the light neutrino or charged lepton masses) to the $T$ parameter are given by [8]:

$$
\alpha T=\frac{\alpha}{8 \pi s_{\mathrm{W}}^{2} M_{W}^{2}}\left(\sum_{\alpha, \beta, i, j}\left(U_{\alpha i}^{*} U_{\alpha j} U_{\beta i} U_{\beta j}^{*} f\left(M_{i}, M_{j}\right)+U_{\alpha i}^{*} U_{\alpha j} U_{\beta i}^{*} U_{\beta j} g\left(M_{i}, M_{j}\right)\right),\right.
$$

and where $M_{i}$ are the neutrino mass eigenvalues.

If $L$ is approximately conserved, the first term in Eq. (4.1), which is always positive, dominates the contribution to $T$. Then $\alpha T \geq 0$ and no cancellation is allowed. Thus, significant violations of $L$ are necessary so that the second term in Eq. (4.1), which is allowed to be negative, can dominate 
over the first. If both $\mu_{1}$ and $\mu_{3}$ are simultaneously included and dominate over the $L$-conserving $\Lambda$ and $\Lambda^{\prime}$ then $T$ is given by:

$$
T \simeq \frac{v_{\mathrm{EW}}^{4}}{64 \pi s_{\mathrm{W}}^{2} M_{W}^{2}}\left(\sum_{\alpha}\left|Y_{\alpha}\right|^{2}\right)^{2} \frac{6 \mu \mu_{1}-\left(3 \mu_{1}^{2}+\mu^{2}\right) \log \left(\frac{\mu+\mu_{1}}{\mu-\mu_{1}}\right)}{\mu^{3} \mu_{1}},
$$

where $\mu=\sqrt{\mu_{1}^{2}+4 \mu_{3}^{2}}$. On the other hand, the loop contributions mediated by $\mu_{1}$ and $\mu_{3}$ to the light neutrino masses are found to be [17, 18]:

$$
\Delta m_{v_{\alpha \beta}}=\frac{Y_{\alpha} Y_{\beta}}{32 \pi^{2} \mu}\left(3 M_{Z}^{2} h\left(M_{Z}\right)+M_{h}^{2} h\left(M_{h}\right)\right) .
$$

These corrections can indeed be sizable and in Fig. 2 we show the values that the loop contribution to the light neutrino masses take in order to recover a given value for $-2 \alpha T$ for different values of $\mu_{1}$ and $\mu_{3}$. If $-2 \alpha T \sim 10^{-3}$ so as to implement the cancellation between tree and loop level contributions, corrections to the light neutrino masses ranging from $\sim 100 \mathrm{keV}$ to $\sim 100 \mathrm{MeV}$ would be obtained, far exceeding present constraints.

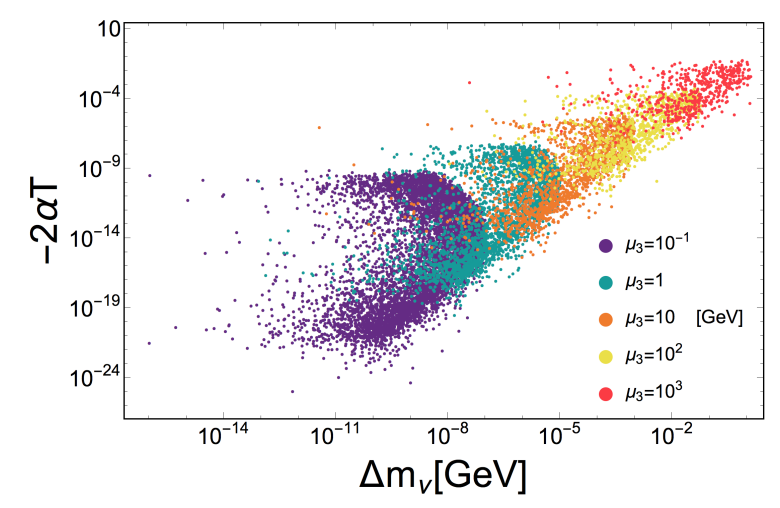

Figure 2: $T$ parameter versus 1-loop correction to $m_{v}$ for different values of the $L$-violating parameters $\mu_{1}$ and $\mu_{3}$.

\section{Conclusions}

We have analyzed in detail the importance of loop corrections when deriving constraints on the mixing between the SM flavour eigenstates and the new heavy neutrinos introduced in Seesaw mechanisms.

We conclude that loop level corrections are only relevant in a small fraction of the Seesaw parameter space characterized by large Yukawa couplings and low Seesaw scale and that these corrections tend to strengthen the tree level contributions unless large deviations from $L$ are present. If $L$ is approximately conserved, data thus prefer regions of the parameter space where these loops are irrelevant. On the other hand, if $L$ is strongly violated, the cancellation discussed in Ref. [8] can indeed provide a good fit to data with a very relevant role of the loop contributions. However, these large violations of $L$ at loop level also lead to too large contributions to the light neutrino masses 
and hence this possibility is ruled out. We therefore conclude that loop corrections can safely be neglected in analyses of the heavy neutrino mixings in Seesaw mechanisms.

Finally we have also obtained relevant constraints on this mixing when $L$ is an approximate symmetry. We find a mild ( $\sim 90 \% \mathrm{CL}$ ) preference for non-zero mixing with the $e$ flavours with a best fit at $\theta_{e}=0.034_{-0.014}^{+0.009}$ for normal mass hierarchy. This preference also induces non-zero mixing with the $\tau$ flavour $\theta_{\tau}=0.018_{-0.013}^{+0.019}$ so as to recover the correct pattern of neutrino masses and mixings. On the other hand, small $\theta_{\mu}$ is preferred so as to keep $\mu \rightarrow e \gamma$ at acceptable levels in presence of non-zero $\theta_{e}$. At the $2 \sigma$ level the following upper bounds are found: $\theta_{e}<0.051$, $\theta_{\mu}<0.037$ and $\theta_{\tau}<0.049$.

\section{Acknowledgments}

I acknowledge financial support by the European Union through the ITN INVISIBLES (PITNGA-2011-289442). I also acknowledge support from the EU through the FP7 Marie Curie Actions CIG NeuProbes (PCIG11-GA-2012-321582) and the Spanish MINECO through the "Ramón y Cajal" programme (RYC2011-07710), the project FPA2009-09017 and through the Centro de excelencia Severo Ochoa Program under grant SEV-2012-0249.

\section{References}

[1] M. Gonzalez-Garcia, M. Maltoni, and T. Schwetz, JHEP 1411, 052 (2014), [1 409.5439 ].

[2] R. Mohapatra and J. Valle, Phys.Rev. D34, 1642 (1986).

[3] J. Bernabeu, A. Santamaria, J. Vidal, A. Mendez, and J. Valle, Phys.Lett. B187, 303 (1987).

[4] G. C. Branco, W. Grimus, and L. Lavoura, Nucl. Phys. B312, 492 (1989).

[5] W. Buchmuller and D. Wyler, Phys.Lett. B249, 458 (1990).

[6] S. Weinberg, Phys.Rev.Lett. 43, 1566 (1979).

[7] M. Malinsky, J. Romao, and J. Valle, Phys.Rev.Lett. 95, 161801 (2005), [hep-ph/0506296].

[8] E. Akhmedov, A. Kartavtsev, M. Lindner, L. Michaels, and J. Smirnov, JHEP 1305, 081 (2013), [1302.1872].

[9] L. Basso, O. Fischer, and J. J. van der Bij, Europhys.Lett. 105, 11001 (2014), [1310 . 2057 ] .

[10] S. Antusch and O. Fischer, JHEP 1410, 94 (2014), [1407.6607] .

[11] E. Fernandez-Martinez, J. Hernandez-Garcia, J. Lopez-Pavon and M. Lucente, [1508.03051] .

[12] M. Blennow and E. Fernandez-Martinez, Phys.Lett. B704, 223 (2011), [1107. 3992 ].

[13] A. Abada, C. Biggio, F. Bonnet, M. B. Gavela, and T. Hambye, JHEP 12, 061 (2007), [0707.4058].

[14] R. Adhikari and A. Raychaudhuri, Phys. Rev. D84, 033002 (2011), [1004.5111].

[15] J. M. Cornwall, D. N. Levin, and G. Tiktopoulos, Phys. Rev. D10, 1145 (1974), [Erratum: Phys. Rev.D11,972(1975)].

[16] K. A. Olive et al. (Particle Data Group), Chin. Phys. C38, 090001 (2014).

[17] A. Pilaftsis, Z. Phys. C55, 275 (1992), [hep-ph/9901206].

[18] J. Lopez-Pavon, S. Pascoli, and C.-f. Wong, Phys.Rev. D87, 093007 (2013), [1209. 5342 ]. 